\title{
Convergent close coupling versus the generalized Sturmian function approach: Wave-function analysis
}

\author{
M. Ambrosio and D. M. Mitnik \\ Instituto de Astronomía y Física del Espacio (IAFE, CONICET-UBA), Casilla de Correo 67, Sucursal 28 (C1428ZAA), \\ Ciudad Autónoma de Buenos Aires, Argentina \\ G. Gasaneo \\ Departamento de Física, Universidad Nacional del Sur, 8000 Bahía Blanca, Buenos Aires, Argentina
}

J. M. Randazzo

División Física Atómica, Molecular y Óptica, Centro Atómico Bariloche, 8400 San Carlos de Bariloche, Río Negro, Argentina

\author{
A. S. Kadyrov, D. V. Fursa, and I. Bray \\ Curtin Institute for Computation and Department of Physics and Astronomy, Curtin University, \\ G.P.O. Box U1987, Perth, Western Australia 6845, Australia \\ (Received 1 September 2015; published 23 November 2015)
}

\begin{abstract}
We compare the physical information contained in the Temkin-Poet (TP) scattering wave function representing electron-impact ionization of hydrogen, calculated by the convergent close-coupling (CCC) and generalized Sturmian function (GSF) methodologies. The idea is to show that the ionization cross section can be extracted from the wave functions themselves. Using two different procedures based on hyperspherical Sturmian functions we show that the transition amplitudes contained in both GSF and CCC scattering functions lead to similar single-differential cross sections. The single-continuum channels were also a subject of the present studies, and we show that the elastic and excitation amplitudes are essentially the same as well.
\end{abstract}

DOI: 10.1103/PhysRevA.92.052518

PACS number(s): 31.15.A-, 31.15.xj

\section{INTRODUCTION}

From the very beginning of quantum mechanics scattering theory has been of great importance due to the direct applications it has in many areas of physics. Of particular interest to us is the few-body context. In 1999, Rescigno et al. [1] using the exterior complex scaling (ECS) proved for the first time that the three-body problem was solvable. Today there are many methods which can solve the quantum three-body problem in addition to the ECS: convergent close coupling (CCC), the $J$ matrix, the $R$ matrix, time-dependent close coupling [2-4], and more recently the generalized Sturmian function approach (GSF) [5]. However, processes such as double ionization of helium by high-energy electron impact show that this problem is not closed or completely understood [6,7], though some benchmark results for the $S$-wave model exist $[8,9]$. At high incident energies the four-body $(e, 3 e)$ problem can be reduced to a three-body one [5]. Even so, within this three-body framework no agreement is found between three independent ab initio methods: CCC [6], the $J$ matrix [10-12], and wave-packet evolution [13].

In the following we focus on time-independent methods. The ECS, CCC, $J$-matrix, and GSF methods solve the threebody Schrödinger equation to a precision that in principle can be arbitrarily increased by just incrementing the grid density or the number of basis functions. The three-body scattering problem requires the imposition of appropriate outgoing type asymptotic conditions which represent the particle emission. For the double continuum those consist of hyperspherical outgoing wave fronts, when all the emitted particles are far apart from each other. The GSF approach imposes outgoing flux to each of the electron coordinates [14]. This is done by using GSFs which are obtained by solving a two-body Schrödinger equation and where the outgoing behavior is explicitly enforced on the solutions. The three-body wave function is proposed as a linear combination of products of GSFs for each electron coordinate. The discussion presented by Ambrosio et al. [15] explains how the GSF method in the spherical coordinates $\left(r_{1}, r_{2}\right)$ can provide a good characterization of a problem whose asymptotic conditions are defined on the hyperspherical coordinate $\rho$.

Both approaches, ECS and GSFs, treat the emitted particles on an equal footing, and in both schemes the resulting wave function is sufficiently accurate to allow the extraction of the transition matrix directly from its asymptotic region.

Methods aimed at obtaining integrated quantities like scattering amplitudes directly rather than through the wave functions, on the other hand, contain particular features that make them very different from ECS and GSFs. In the CCC framework, see Ref. [16] for a recent review of applications to ionization problems; the electrons are treated in an asymmetric manner. For instance, in the ionization of hydrogen by electron impact, CCC uses for one of the electrons an $\mathcal{L}^{2}$ basis and for the second one uses stationary target continuum states. The target (pseudo) states are obtained by diagonalization of the target Hamiltonian in a Laguerre basis [17] or are box based [18]. Due to the standing wave-type behavior imposed on the basis, the spectra of the target are not only discrete and finite (for a finite number of basis functions) but also real. So no outgoing-type behavior is imposed on the target system. The three-body outgoing behavior is in principle guaranteed by the use of the three-body Green's function in the formulation 
of the method. However, it has never been shown that the method effectively generates the outgoing hyperspherical wave front expected for the three-body double continuum. Additionally, it was found that the cross sections obtained present oscillations around the correct shape and a stepfunction behavior near the equal-energy-sharing point $[19,20]$. The asymmetric treatment of the two electrons leads to an asymmetric single-differential cross section (SDCS), even though symmetrization is included and the results are obtained independently for a total spin $S$. Various discussions have been presented to understand this behavior [21-23] within the electron-hydrogen Temkin-Poet (TP) framework [24,25] for which the calculations are much simpler, due to the absence of any angular momentum algebra. This is an ideal model for developing general scattering theories and testing numerical approaches. The original CCC method was tested on this TP model [26], which continues to be of great importance for further development of the method [27], and its relation to other approaches.

In this paper we study these issues by performing a comparison between the results obtained with the GSF and CCC methods. We explicitly show that the reconstructed CCC total wave function indeed contains the expected outgoing hyperspherical wave fronts. Furthermore, we show that the CCC scattering wave function can be used to directly extract the transition amplitude and the cross sections, as done with the GSF [5]. In fact, the SDCS extracted from the CCC wave function this way does not display any step-function behavior. Atomic units $(\hbar=e=1)$ are assumed throughout.

\section{STATEMENT OF THE THREE-BODY PROBLEM}

Let us first define the problem to be analyzed. We start with the Schrödinger equation corresponding to the electron impact ionization of hydrogen (nuclear charge $Z=1$ ) in the TP model [24,25]:

$$
\begin{gathered}
{\left[-\frac{1}{2 r_{1}^{2}} \frac{\partial}{\partial r_{1}}\left(r_{1}^{2} \frac{\partial}{\partial r_{1}}\right)-\frac{1}{2 r_{2}^{2}} \frac{\partial}{\partial r_{2}}\left(r_{2}^{2} \frac{\partial}{\partial r_{2}}\right)\right.} \\
\left.-\frac{Z}{r_{1}}-\frac{Z}{r_{2}}+\frac{1}{r_{>}}-E\right] \Psi\left(r_{1}, r_{2}\right)=0 .
\end{gathered}
$$

Here $r_{1}$ and $r_{2}$ are the electron coordinates, and $E$ is the total energy of the three-body system. The scattering process develops from the initial asymptotic state, i.e., the hydrogen ground state and a plane wave of incident momentum $\mathbf{k}_{i}$. In a time-independent representation this implies the definition of the initial channel:

$$
\Psi_{0}\left(r_{1}, r_{2}\right)=\frac{1}{\sqrt{2}}\left[j_{0}\left(k_{i} r_{1}\right) \phi\left(r_{2}\right)+(-1)^{S} j_{0}\left(k_{i} r_{2}\right) \phi\left(r_{1}\right)\right] .
$$

We search for the solution of the Schrödinger equation describing the full dynamic of the process. This can be written as

$$
\Psi^{+}\left(r_{1}, r_{2}\right)=\Psi_{0}\left(r_{1}, r_{2}\right)+\Psi_{\mathrm{sc}}^{+}\left(r_{1}, r_{2}\right)
$$

The function $\Psi_{\mathrm{sc}}^{+}\left(r_{1}, r_{2}\right)$ contains all of the information about the scattering process and the correlation between the particles. Substituting $\Psi^{+}\left(r_{1}, r_{2}\right)$, of Eq. (3), in Eq. (1) we obtain the following driven equation:

$$
\begin{aligned}
& {\left[-\frac{1}{2 r_{1}^{2}} \frac{\partial}{\partial r_{1}}\left(r_{1}^{2} \frac{\partial}{\partial r_{1}}\right)-\frac{1}{2 r_{2}^{2}} \frac{\partial}{\partial r_{2}}\left(r_{2}^{2} \frac{\partial}{\partial r_{2}}\right)\right.} \\
& \left.-\frac{Z}{r_{1}}-\frac{Z}{r_{2}}+\frac{1}{r_{>}}-E\right] \Psi_{\mathrm{sc}}^{+}\left(r_{1}, r_{2}\right)=\mathcal{F}\left(r_{1}, r_{2}\right) .
\end{aligned}
$$

The source term $\mathcal{F}\left(r_{1}, r_{2}\right)$ is

$$
\begin{aligned}
\mathcal{F}\left(r_{1}, r_{2}\right)= & \frac{1}{\sqrt{2}}\left(-\frac{Z}{r_{1}}+\frac{1}{r_{>}}\right) j_{0}\left(k_{i} r_{1}\right) \phi\left(r_{2}\right) \\
& +\frac{1}{\sqrt{2}}\left(-\frac{Z}{r_{2}}+\frac{1}{r_{>}}\right)(-1)^{S} j_{0}\left(k_{i} r_{2}\right) \phi\left(r_{1}\right) .
\end{aligned}
$$

The description of hydrogen ionization requires solving the driven equation (4) with the appropriate asymptotic conditions. Within the TP model, this means enforcing on $\Psi_{\mathrm{sc}}^{+}\left(r_{1}, r_{2}\right)$ at large distances the following form:

$$
\Psi_{\mathrm{sc}}^{+}\left(r_{1}, r_{2}\right) \rightarrow \mathcal{T}(E, \alpha) \frac{e^{i\left[K \rho-\frac{C(\alpha)}{K} \ln (2 K \rho)\right]}}{\rho^{\frac{5}{2}}} .
$$

Here $\rho=\sqrt{r_{1}^{2}+r_{2}^{2}}$ and $\alpha=\arctan \left(r_{2} / r_{1}\right)(\alpha \in[0, \pi / 2])$ are the hyperradius and hyperangle, respectively, and $K=\sqrt{2 E}$ is the hyperspherical momentum. $C(\alpha)$ defines an angular dependent charge and it is given by

$$
C(\alpha)=-\frac{Z}{\cos \alpha}-\frac{Z}{\sin \alpha}+ \begin{cases}\frac{1}{\cos \alpha}, & 0 \leqslant \alpha \leqslant \frac{1}{4} \pi, \\ \frac{1}{\sin \alpha}, & \frac{1}{4} \pi \leqslant \alpha \leqslant \frac{1}{2} \pi .\end{cases}
$$

In Eq. (6) $\mathcal{T}(E, \alpha)$ represents the transition amplitude for the ionization process.

Equation (1) shows that the radial coordinates are coupled through the interaction $1 / r_{>}$. From a practical point of view, imposing the asymptotic behavior (6) on the scattering solution $\Psi_{\mathrm{sc}}^{+}\left(r_{1}, r_{2}\right)$ is the key and is a difficult problem.

\section{WAVE-FUNCTION COMPARISON}

In this section we obtain and compare the wave functions calculated with the GSF and CCC methods. It is helpful to work with the reduced function

$$
\begin{aligned}
\Phi_{\mathrm{sc}}^{+}\left(r_{1}, r_{2}\right) & =r_{1} r_{2} \Psi_{\mathrm{sc}}^{+}\left(r_{1}, r_{2}\right) \\
& \equiv \rho^{2} \sin (\alpha) \cos (\alpha) \Psi_{\mathrm{sc}}^{+}(\rho, \alpha) \\
& \equiv \Phi_{\mathrm{sc}}^{+}(\rho, \alpha),
\end{aligned}
$$

where for brevity of notation we make the dependence on the total spin $S$ implicit. Without loss of generality we take $54.4-\mathrm{eV}$ electrons incident on the ground state of atomic hydrogen, within the TP model where only states with zero orbital angular momenta are retained. At this energy the total ionization cross section is near its maximum for both $S=0$ and 1 (see, for example, Fig. 3 of Bray et al. [27]). In the CCC calculations we have taken 30 Laguerre functions with an exponential falloff [17] $\lambda=2$, i.e., optimized for the ground state. In the CCC method the solution of the coupled equations yields the scattering transition amplitudes $T_{S}$ directly, with the variational principle assisting in their accuracy. It is unusual to reconstruct the wave function $\Psi^{+}\left(r_{1}, r_{2}\right)$ in coordinate space, but we do so when calculating double photoionization [20,28], 

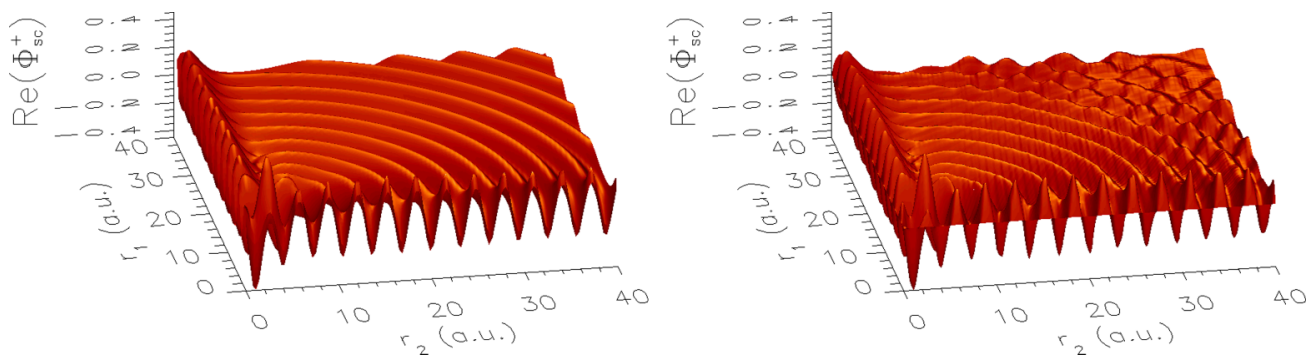

FIG. 1. (Color online) Real part of the scattering wave functions, see Eq. (8), for the singlet case of 54.4-eV electron scattering on atomic hydrogen in the TP model. The present GSF results are on the left and the CCC ones are on the right.

and we do so here for the one-dimensional case. Having solved the CCC equations [17] for the transition amplitudes via

$$
\begin{aligned}
\left\langle k_{f} \phi_{f}\left|T_{S}\right| \phi_{i} k_{i}\right\rangle= & \left\langle k_{f} \phi_{f}\left|V_{S}\right| \phi_{i} k_{i}\right\rangle+\sum_{n=1}^{N} \\
& \times \int_{0}^{\infty} \frac{d k\left\langle k_{f} \phi_{f}\left|V_{S}\right| \phi_{n} k\right\rangle\left\langle k \phi_{n}\left|T_{S}\right| \phi_{i} k_{i}\right\rangle}{E+i 0-\epsilon_{n}-k^{2} / 2}
\end{aligned}
$$

we write

$$
\begin{aligned}
\Psi^{+}\left(r_{1}, r_{2}\right)= & \left\langle r_{1} r_{2} \mid \phi_{i} k_{i}\right\rangle \\
& +\sum_{n=1}^{N} \int_{0}^{\infty} \frac{d k\left\langle r_{1} r_{2} \mid \phi_{n} k\right\rangle\left\langle k \phi_{n}\left|T_{S}\right| \phi_{i} k_{i}\right\rangle}{E+i 0-\epsilon_{n}-k^{2} / 2} .
\end{aligned}
$$

A different path is taken within the GFS approach [14,29-31], since we work our way up to the transition amplitudes starting with the wave-function calculation, from Eq. (4). The not yet determined solution is proposed as a linear combination of a product of two-body GSF functions:

$$
\begin{aligned}
\Psi_{\mathrm{sc}}^{+}\left(r_{1}, r_{2}\right)= & \sum_{n_{1}, n_{2}} a_{n_{1}, n_{2}}^{S} g_{n_{1}, n_{2}} \frac{1}{r_{1} r_{2}} \frac{1}{\sqrt{2}}\left[S_{n_{1}}^{+}\left(r_{1}\right) S_{n_{2}}^{+}\left(r_{2}\right)\right. \\
& \left.+(-1)^{S} S_{n_{1}}^{+}\left(r_{2}\right) S_{n_{2}}^{+}\left(r_{1}\right)\right]
\end{aligned}
$$

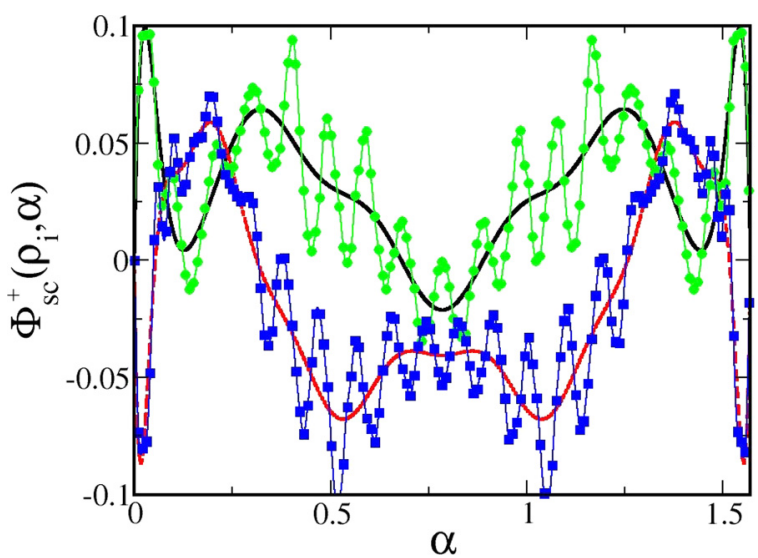

FIG. 2. (Color online) Example of the filtering process. Black solid line: Filtered CCC real. Red dashed line: Filtered CCC imaginary. Green line with circles: Unfiltered CCC real. Blue line with squares: Unfiltered CCC imaginary. Hyperradius: $\rho=36.37$ a.u.. where $a_{n_{1}, n_{2}}^{S}$ are linear coefficients for a given spin state $S$ (singlet or triplet) and the factor $g_{n_{1}, n_{2}}=\left[1-\delta_{n_{1}, n_{2}}\left(1-\frac{1}{\sqrt{2}}\right)\right]$ ensures no double counting is done in the symmetrization. After inserting the expansion (11) into Eq. (4), and projecting on the left with the GSF, the driven equation is transformed into a linear system of equations:

$$
[H-E \mathcal{O}] \mathbf{a}=\mathbf{f},
$$

where the matrix $\mathcal{O}$ contains the overlaps between the threebody GSF basis elements appearing in Eq. (11). The matrix $H$ is the GSF representation of the full Hamiltonian [see Ref. [14], Eq. (45)]. Once the wave function is obtained, the collisional information is extracted from it [5,30,32]. For the present kinematics, we used 50 Sturmians per coordinate.

We begin by displaying the resulting solutions from Eq. (4) for $S=0$ in Fig. 1. Only the real parts are shown, since the imaginary parts have similar structures. Both methods yield the asymptotic behavior required by the Peterkop asymptotic conditions [33,34], with the GSF results going out accurately to larger distances. Beyond a given hyperradius $(\rho \approx 35)$, the usage of the short-ranged Laguerre basis limits the quality of the CCC-calculated wave function.

\section{SINGLE-DIFFERENTIAL CROSS SECTION EXTRACTION}

While both wave functions contain essentially the same double-continuum physics, from Fig. 1 it is clear that the GSF solution is more accurate. We wish to extract the SDCS from the asymptotic range of both wave functions (GSF and CCC), based on the work by Kadyrov et al. [34,35]. In terms of the

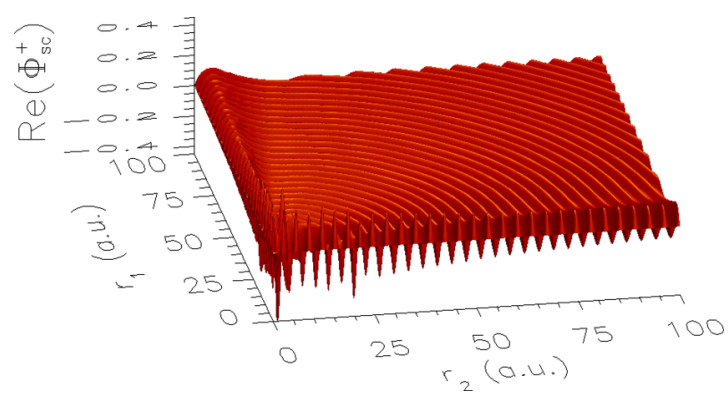

FIG. 3. (Color online) Real part of the $(e, 2 e)$ scattering function, obtained by the GSF method but extended via the HGSF expansion. 


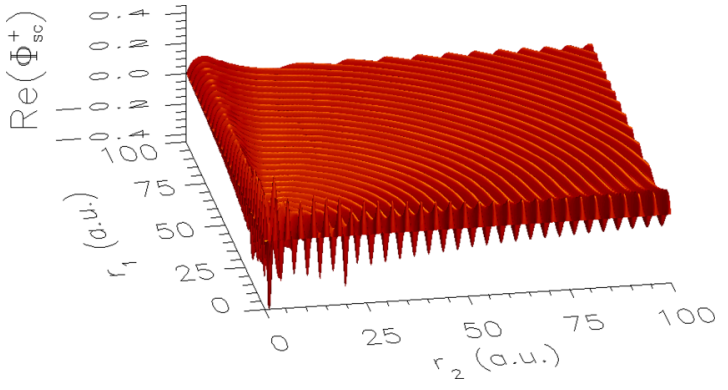

FIG. 4. (Color online) Real part of the $(e, 2 e)$ scattering function, obtained by the CCC method but filtered and extended via the HGSF expansion.

reduced function (8) for the TP model, the SDCS is written as

$$
\frac{d \sigma(\alpha)}{d E_{2}}=\frac{1}{k_{i} K} \lim _{\rho \rightarrow \infty} \frac{\rho\left|\Phi_{\mathrm{sc}}^{+}(\rho, \alpha)\right|^{2}}{\sin (\alpha) \cos (\alpha)} .
$$

\section{Filtering procedure for the $\mathrm{CCC}$ solution}

As can be seen in Fig. 1, the CCC function presents a mild degree of noise, which would propagate to the cross section if Eq. (13) were used directly. To filter the CCC wave function we reexpand it in terms of a basis set in the hyperangular coordinate $\alpha$, which we refer to as hyperangular Sturmian functions (HaSFs). These functions, denoted by $H_{n}(\alpha)$, satisfy the following:

$$
-\frac{1}{2} \frac{d^{2}}{d \alpha^{2}} H_{n}(\alpha)=-\beta_{n}\left(\frac{Z_{\cos }}{\cos (\alpha)}+\frac{Z_{\sin }}{\sin (\alpha)}+Z_{0}\right) H_{n}(\alpha),
$$
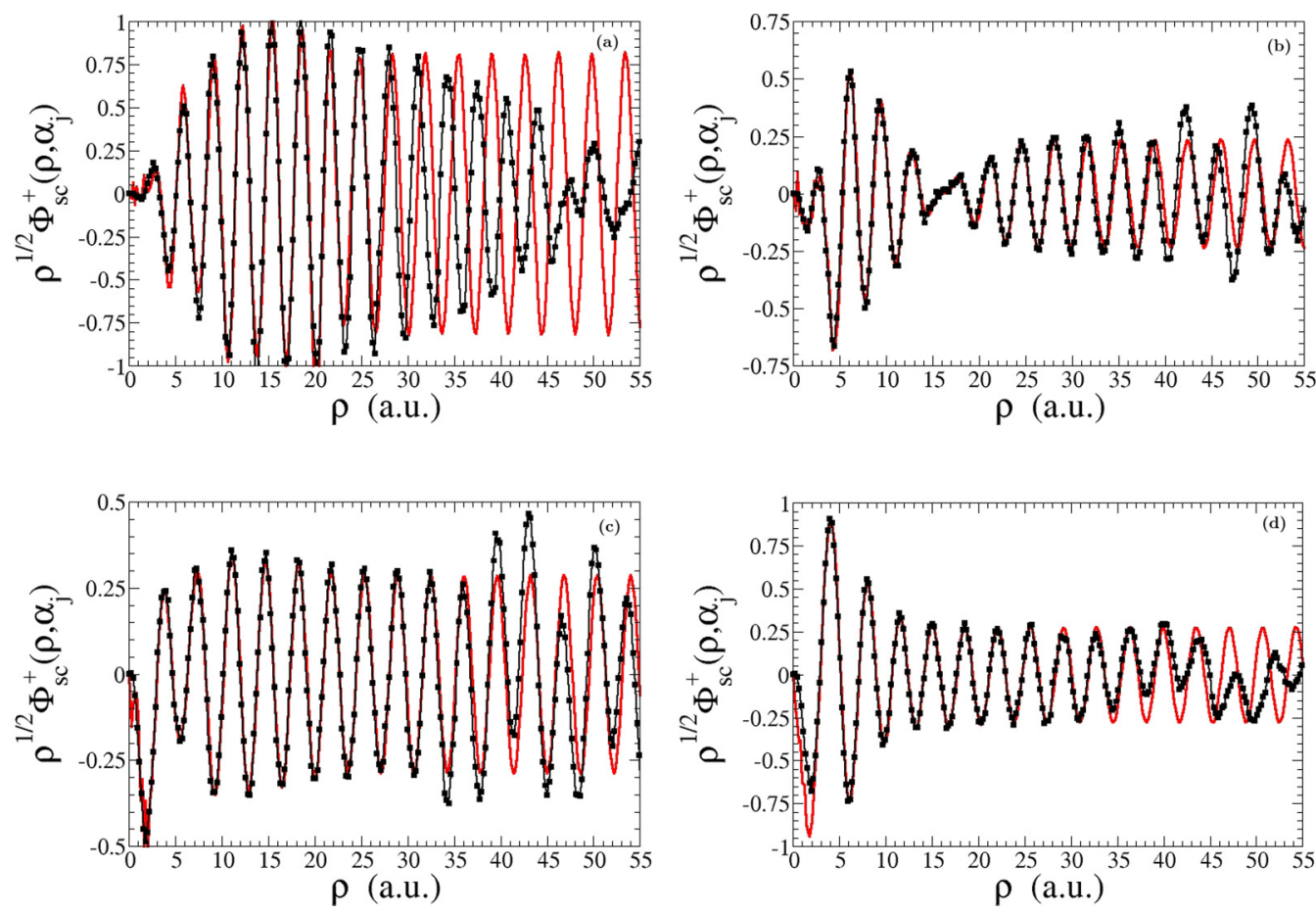

FIG. 5. (Color online) Real part of the (e,2e) scattering function, fixed hyperangle $\alpha_{j}$ cuts: (a) $\alpha_{1}=5^{\circ}$, (b) $\alpha_{2}=15^{\circ}$, (c) $\alpha_{3}=30^{\circ}$, and (d) $\alpha_{4}=45^{\circ}$. Original: Black solid line with squares. Reexpanded: Solid red line. 

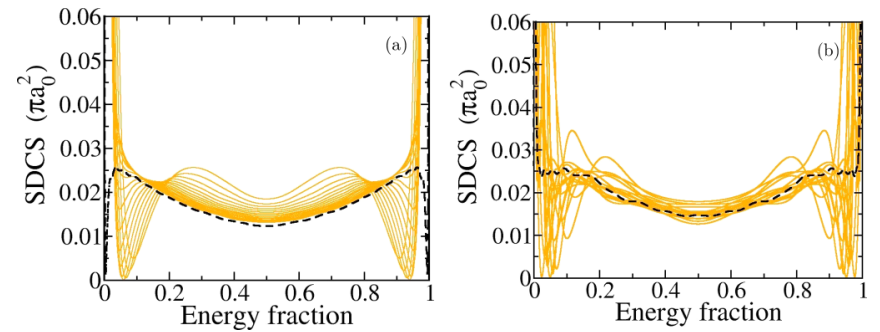

FIG. 6. (Color online) SDCS. (a) GSF. (b) CCC. The orange lines show the SDCSs calculated via interpolation and filtering of fixed hyperradial cuts. The black dashed line shows the SDCS extracted from the HGSF reexpansion.

\section{REEXPANSION PROCEDURE WITH A HYPERSPHERICAL OUTGOING BASIS SET}

For the purpose of extracting the transition amplitude, we reexpanded the CCC and GSF solutions in terms of a hyperspherical Sturmian basis. Hyperspherical generalized Sturmian functions (HGSFs) were introduced by Gasaneo et al. [36]. For application examples, see Refs. [5,37].

The three-body basis is composed of outer products of noncoupled hyperangular and hyperradial functions. The hyperradial functions satisfy the following differential equation:

$$
\begin{aligned}
& {\left[-\frac{1}{2 \mu}\left(\frac{d^{2}}{d \rho^{2}}-\frac{l(l+4)+15 / 4}{\rho^{2}}\right)+\mathcal{U}(\rho)-E_{s}\right] \mathcal{S}_{n, l}(\rho)} \\
& \quad=-\beta_{n, l} \mathcal{V}(\rho) \mathcal{S}_{n, l}(\rho),
\end{aligned}
$$

with energy $E_{s}$ and potential $\mathcal{U}(\rho)$ shaping the asymptotic behavior of the basis. The generally short-ranged potential $\mathcal{V}(\rho)$ regulates where the oscillations of the basis set are mostly localized. In this particular application we $\operatorname{chose} \mathcal{U}(\rho)=-\frac{\sqrt{2}}{\rho}$, whose asymptotic charge is exact for $\alpha=\pi / 4$ [see Eq. (7)], and a Woods-Saxon type $\mathcal{V}(\rho)$. Angular functions are used to expand the logarithmic phase for other hyperangles. We chose an energy coincident with the total available energy for the electrons, i.e., $E_{s}=E_{a}=1.5$ a.u. Both the parameters $E_{s}$ and the charge in $\mathcal{U}(r)$ are of fundamental importance for the radial HGSF. If they are properly selected, not many HGSF basis elements are required because the basis has the correct asymptotic behavior built in.

We construct a hyperspherical three-body basis with a radial HGSF multiplied by the $H_{n}(\alpha)$ (HaSF) already defined in
Eq. (14),

$$
\Theta_{\nu}(\rho, \alpha)=\mathcal{S}_{n, l}(\rho) H_{m}(\alpha)
$$

and with this the expansion reads

$$
\Phi_{\mathrm{sc}}^{+}\left(r_{1}, r_{2}\right)=\frac{1}{\sqrt{\rho}} \sum_{n, m} a_{n, m} \Theta_{\nu}(\rho, \alpha) .
$$

We stress that the HGSF basis is used to reexpand the already determined scattering GSF and CCC functions. The idea is to take advantage of a readily extractable transition amplitude, since the basis already has the expected hyperspherical outgoing behavior that factors out from Eq. (6). We use a small number of basis elements to filter the higher-frequency angular oscillations present mainly in the CCC wave function. In expansion (18) we worked with as few as 20 angular and 15 radial functions, since the purpose was to extract the chief features of the double continuum and filter out the unwanted lateral wrinkles.

For this application, we found that the best way to obtain a good representation of $\Psi_{\mathrm{sc}}^{+}\left(r_{1}, r_{2}\right)$ at least far from the origin consists of projecting Eq. (18) onto every basis element as

$$
\begin{aligned}
& \int d r_{1} d r_{2} \Theta_{\nu^{\prime}}^{*}(\rho, \alpha) \rho^{1 / 2} \Phi_{\mathrm{sc}}^{+}\left(r_{1}, r_{2}\right) \\
& =\sum_{n, m} a_{n, m} \int d r_{1} d r_{2} \Theta_{\nu^{\prime}}^{*}(\rho, \alpha) \Theta_{v}(\rho, \alpha),
\end{aligned}
$$

where the asterisk denotes complex conjugation. This differs from the standard usage of the HGSF basis presented in Ref. [5], but this worked best for the present analysis. We chose to perform the integrations in Eq. (19) using the $\left(r_{1}, r_{2}\right)$ coordinates. Consequently, we used a modified bilinear scheme to translate the hyperspherical basis into the rectangular grid, maintaining good accuracy. With the whole integrand expressed in $\left(r_{1}, r_{2}\right)$ coordinates, a two-dimensional Simpson integration was applied. The integration boundaries were controlled via a masking function, which smoothly shut off the integrand beyond a given hyperradius, $\rho_{\max }$. The two-dimensional integration was, for the CCC case, restricted to the region inside $\rho<\rho_{\max }=30$ a.u., in order to avoid the less desirable regions where the unphysical oscillations begin to appear.

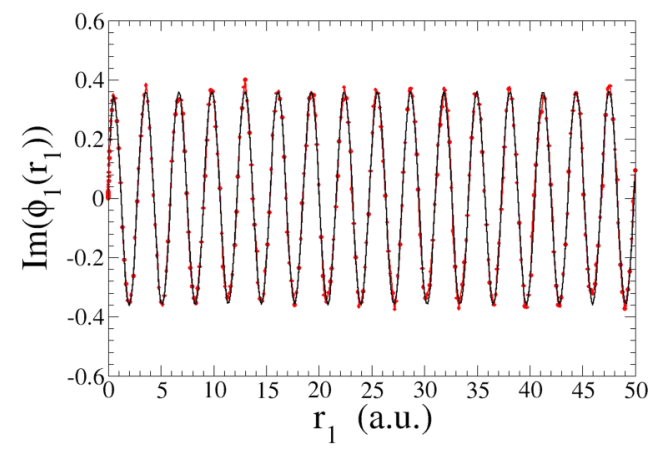

FIG. 7. (Color online) Elastic channel, real part (left panel) and imaginary part (right panel). GSF: Black solid line. CCC: Red line with circles. 

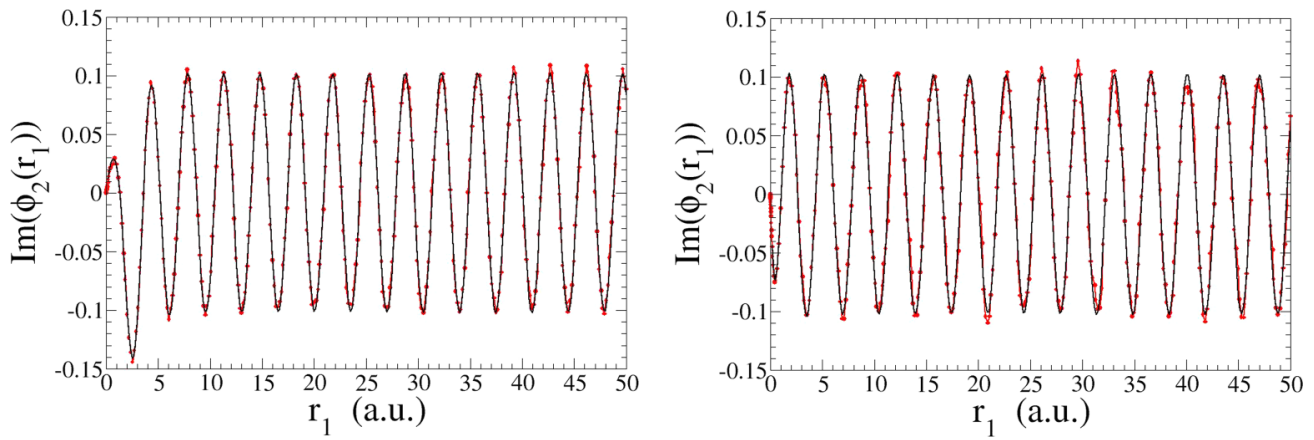

FIG. 8. (Color online) $n=2$ excitation channel, real part (left panel) and imaginary part (right panel). GSF: Solid black line. CCC: Red line with circles.

Equation (19) can adopt a matrix form when run through the indices $m, n$ and $n^{\prime}, m^{\prime}$ :

$$
\mathbf{S} \mathbf{a}=\mathbf{b}
$$

with b collecting the terms from the integral with $\Phi_{\mathrm{sc}}^{+}\left(r_{1}, r_{2}\right)$. This resulting linear system in $a_{n, m}$ is solved numerically with the routine ZGESV from the LAPACK [38] library.

We show in Figs. 3 and 4 the real parts of the scattering wave functions in their reexpanded versions. The HGSF basis is tailored to efficiently expand the double-continuum region. To some degree, the single continuum is also sketched, but this comes more as a by-product.

To show that our expansion does match the original functions properly in the region where they are well defined, we show in Fig. 5 some fixed hyperangle cuts of the original CCC function and its reexpanded counterpart. Figure 5(a) shows less agreement because of the low hyperangle, which implies that the cut passes through a sector where the single continuum (see the shorter wavelengths) is dominant, whereas the reexpansion is tailored to match the double-continuum wave fronts.

By virtue of expressions (18) and (6) the squared module of the transition amplitude is simply

$$
|\mathcal{T}(\alpha)|^{2}=\left|\sum_{n, m} a_{n, m} \Theta\left(\rho_{\mathrm{as}}, \alpha\right)\right|^{2},
$$

where $\rho_{\text {as }}$ is any hyperradius beyond the range of $\mathcal{V}(\rho) \mathcal{S}_{n, l}(\rho)$.

It is of interest to note that the calculation of the GSF function with that degree of precision requires about 2500 basis elements, whereas the HGSF reexpansion was acceptable

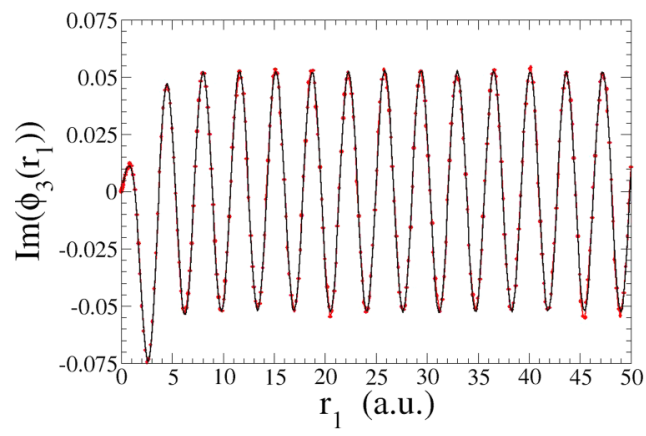

in the far region, requiring only 300 elements. Clearly, the HSGF approach shows strong potential for double-continuum problems.

\section{SDCS results}

We proceed to compare the SDCSs that originate from both scattering wave functions and from the two separate procedures: the full reexpansion in terms of the HGSF basis and the angular filtering of the fixed hyperradial cuts via the angular $H_{n}(\alpha)$ basis. Having removed much of the CCC wave-function noise, we proceed to compare its SDCS with the GSF one. Within the double-continuum region, both CCC and GSF point to the same physical content - the cross section, which is an observable quantity. No attempt was made to deal with the behavior of the SDCS in the energy sharing extremes of 0 and 1 .

Two different aspects stand out when observing Figs. 6(a) and 6(b). It is clear that the two different methods for extracting SDCSs provide equivalent results, ensuring that both GSF and CCC lead to similar physical results. The contributions to the SDCS from single-continuum channels are severely diminished in the HGSF representation.

\section{SINGLE-CONTINUUM CHANNELS}

We now turn to the comparison of the single-continuum information contained in both the original wave functions. We obtain the single-continuum functions $\phi_{n}\left(r_{1}\right)$ from $\Phi_{\mathrm{sc}}^{+}\left(r_{1}, r_{2}\right)$ as follows:

$$
\phi_{n}\left(r_{1}\right)=\int_{0}^{\tilde{R}_{2}} d r_{2} \Phi_{\mathrm{sc}}^{+}\left(r_{1}, r_{2}\right) \varphi_{n}\left(r_{2}\right)
$$

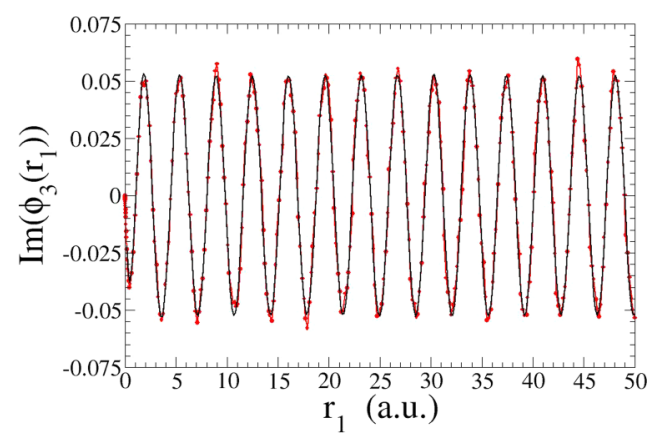

FIG. 9. (Color online) $n=3$ excitation channel, real part (left panel) and imaginary part (right panel). GSF: Solid black line. CCC: Red line with circles. 
TABLE I. Single-continuum amplitudes, GSF calculation.

\begin{tabular}{|c|c|c|c|c|c|c|}
\hline \multirow[b]{2}{*}{$n$} & \multicolumn{3}{|c|}{ GSF } & \multicolumn{3}{|c|}{$\mathrm{CCC}$} \\
\hline & $A b s\left(A_{+}\right)$ & $A b s\left(A_{0}\right)$ & $\operatorname{Abs}\left(A_{0}\right) / A b s\left(A_{+}\right)$ & $A b s\left(A_{+}\right)$ & $\operatorname{Abs}\left(A_{0}\right)$ & $A b s\left(A_{0}\right) / A b s\left(A_{+}\right)$ \\
\hline 1 & 0.3615 & $7.124 \times 10^{-3}$ & 0.01970 & 0.3625 & $6.318 \times 10^{-3}$ & 0.017 \\
\hline 2 & 0.1017 & $3.853 \times 10^{-4}$ & 0.00379 & 0.1027 & $2.349 \times 10^{-3}$ & 0.023 \\
\hline 3 & $5.2572 \times 10^{-2}$ & $1.257 \times 10^{-4}$ & 0.00239 & $5.2577 \times 10^{-2}$ & $1.2570 \times 10^{-4}$ & 0.00239 \\
\hline
\end{tabular}

with $\tilde{R}_{2}$ being the extreme of the radial grid $r_{2}$ considered. States $\varphi_{n}\left(r_{2}\right)$ are simply the target eigenstates. The elastic channels in the CCC and GSF approaches are in good agreement (see Fig. 7).

A similar agreement is observed in Figs. 8 and 9, suggesting that, as expected, the CCC and GSF three-body functions contain the exact same information regarding the excitation channels as well.

We now perform an asymptotic decomposition of the functions $\phi_{n}\left(r_{1}\right)$. This allows us to test whether the solutions are properly calculated and represent the physical situation. We chose to decompose the solutions into an outgoing wave plus a standing wave (another viable choice would have been decomposing the single-continuum functions into outgoing and incoming waves). The coefficients of the expansion represent the amplitudes of the outgoing and stationary waves and are written as, respectively,

$$
\begin{aligned}
& A_{+}=\frac{\phi_{n}\left(R_{1}\right) F\left(Z_{\mathrm{as}}, E_{n}, R_{2}\right)-\phi_{n}\left(R_{2}\right) F\left(Z_{\mathrm{as}}, E_{n}, R_{1}\right)}{H^{+}\left(Z_{\mathrm{as}}, E_{n}, R_{1}\right) F\left(Z_{\mathrm{as}}, E_{n}, R_{2}\right)-F\left(Z_{\mathrm{as}}, E_{n}, R_{1}\right) H^{+}\left(Z_{\mathrm{as}}, E_{n}, R_{2}\right)}, \\
& A_{0}=\frac{\phi_{n}\left(R_{2}\right) H^{+}\left(Z_{\mathrm{as}}, E_{n}, R_{1}\right)-\phi_{n}\left(R_{1}\right) H^{+}\left(Z_{\mathrm{as}}, E_{n}, R_{2}\right)}{H^{+}\left(Z_{\mathrm{as}}, E_{n}, R_{1}\right) F\left(Z_{\mathrm{as}}, E_{n}, R_{2}\right)-F\left(Z_{\mathrm{as}}, E_{n}, R_{1}\right) H^{+}\left(Z_{\mathrm{as}}, E_{n}, R_{2}\right)} .
\end{aligned}
$$

The functions $F\left(Z_{\text {as }}, E_{n}, r\right)$ and $H^{+}\left(Z_{\text {as }}, E_{n}, r\right)$ are the regular and outgoing Coulomb radial functions and are evaluated at $R_{1}$ and $R_{2}$ far enough from the origin. We find that the amplitude extraction is very sensitive to the charges $Z_{\text {as }}$ chosen. In the present case, since one electron remains close to the nucleus, the continuum one sees no net charge $\left(Z_{\text {as }}=0\right)$.

Table I has the outgoing and stationary amplitudes. GSF and $\mathrm{CCC}$ functions contain only minute traces of stationary solution, which cannot be solely ascribed to the three-body functions themselves, but also to the amplitude calculation. In any case, it is clear that the single-continuum channels characterized by both methods are asymptotically outgoing waves, and the single-continuum amplitudes obtained from both schemes agree well.

\section{SUMMARY}

We showed that it is possible to extract the scattering information from the asymptotic GSF- and CCC-calculated wave functions for the $S$-wave model corresponding to hydrogen ionization. The GSF solution is sufficiently accurate, but the presented CCC wave function required a smoothing procedure based on a reexpansion in terms of a hyperangular basis set on specific hyperradii. Those fixed hyperradii arcs were used to calculate the SDCS from the assumed asymptotic range of the wave function. A three-body hyperspherical basis was constructed with hyperangular Sturmian functions and hyperradial generalized Sturmian functions. The CCC and GSF scattering functions were reexpanded with a HGSF basis. The expansion was focused mainly in the region where the double continuum is dominant, i.e., where both radial coordinates are far enough from their respective origins. The asymptotic behavior imposed on the HGSF basis provided an adequate description of the hyperspherical double continuum. We have also considered the single-continuum channels and found that both CCC and GSF scattering functions contain essentially the same excitation and elastic scattering amplitudes.

\section{ACKNOWLEDGMENTS}

We acknowledge the support of the PIP 201301/607 CONICET (Argentina). One of the authors (G.G.) is grateful for the support of the PGI (24/F059) of the Universidad Nacional del Sur. The support of the Australian Research Council, the National Computer Infrastructure, and the Pawsey Supercomputer Centre are gratefully acknowledged.
[1] T. N. Rescigno, C. W. McCurdy, W. A. Isaacs, and M. Baertschy, Phys. Rev. A 60, 3740 (1999).

[2] M. S. Pindzola, D. Mitnik, and F. Robicheaux, Phys. Rev. A 59, 4390 (1999).

[3] M. S. Pindzola and F. J. Robicheaux, Phys. Rev. A 61, 052707 (2000).
[4] M. S. Pindzola, S. A. Abdel-Naby, J. Colgan, and A. Dorn, J. Phys. B 45, 215208 (2012).

[5] G. Gasaneo, D. M. Mitnik, J. M. Randazzo, L. U. Ancarani, and F. D. Colavecchia, Phys. Rev. A 87, 042707 (2013).

[6] A. Kheifets, I. Bray, A. Lahmam-Bennani, A. Duguet, and I. Taouil, J. Phys. B 32, 5047 (1999). 
[7] L. U. Ancarani, C. D. Cappello, and G. Gasaneo, J. Phys.: Conf. Ser. 212, 012025 (2010).

[8] P. L. Bartlett and A. T. Stelbovics, Phys. Rev. A 81, 022715 (2010).

[9] P. L. Bartlett and A. T. Stelbovics, Phys. Rev. A 81, 022716 (2010).

[10] V. A. Knyr, V. V. Nasyrov, and Y. V. Popov, AIP Conf. Proc. 697, 76 (2003).

[11] A. D. Alhaidari, E. J. Heller, H. A. Yamani, and M. S. Abdelmonem, The J-Matrix Method: Developments and Applications, 1st ed. (Springer, New York, 2008).

[12] M. S. Mengoue, M. G. K. Njock, B. Piraux, Y. V. Popov, and S. A. Zaytsev, Phys. Rev. A 83, 052708 (2011).

[13] V. V. Serov, V. L. Derbov, B. B. Joulakian, and S. I. Vinitsky, Phys. Rev. A 75, 012715 (2007).

[14] G. Gasaneo, L. U. Ancarani, D. M. Mitnik, J. M. Randazzo, A. L. Frapiccini, and F. D. Colavecchia, Adv. Quantum Chem. 67, 153 (2013).

[15] M. J. Ambrosio, F. D. Colavecchia, D. M. Mitnik, G. Gasaneo, and L. U. Ancarani, J. Phys.: Conf. Ser. 601, 012004 (2015).

[16] I. Bray, D. V. Fursa, A. S. Kadyrov, A. T. Stelbovics, A. S. Kheifets, and A. M. Mukhamedzhanov, Phys. Rep. 520, 135 (2012).

[17] I. Bray and A. T. Stelbovics, Phys. Rev. A 46, 6995 (1992).

[18] I. Bray, K. Bartschat, and A. T. Stelbovics, Phys. Rev. A 67, 060704(R) (2003).

[19] I. Bray, Phys. Rev. Lett. 78, 4721 (1997).

[20] I. Bray, D. V. Fursa, A. S. Kheifets, and A. T. Stelbovics, J. Phys. B 35, R117 (2002).

[21] I. Bray, Phys. Rev. A 60, 5118 (1999).

[22] M. S. Pindzola, D. Mitnik, and F. Robicheaux, Phys. Rev. A 62, 062718 (2000).
[23] K. Bartschat, M. P. Scott, P. G. Burke, T. Stitt, N. S. Scott, A. N. Grum-Grzhimailo, S. Riordan, G. Ver Steeg, and S. I. Strakhova, Phys. Rev. A 65, 062715 (2002).

[24] A. Temkin, Phys. Rev. 126, 130 (1962).

[25] R. Poet, J. Phys. B 11, 3081 (1978).

[26] I. Bray and A. T. Stelbovics, Phys. Rev. Lett. 69, 53 (1992).

[27] A. W. Bray, I. Abdurakhmanov, A. S. Kadyrov, D. V. Fursa, and I. Bray [Comput. Phys. Commun. (to be published, 2015)].

[28] A. S. Kheifets and I. Bray, Phys. Rev. A 54, R995 (1996).

[29] A. L. Frapiccini, J. M. Randazzo, G. Gasaneo, and F. D. Colavecchia, J. Phys. B 43, 101001 (2010).

[30] J. M. Randazzo, F. Buezas, A. L. Frapiccini, F. D. Colavecchia, and G. Gasaneo, Phys. Rev. A 84, 052715 (2011).

[31] D. M. Mitnik, F. D. Colavecchia, G. Gasaneo, and J. M. Randazzo, Comput. Phys. Commun. 182, 1145 (2011).

[32] M. J. Ambrosio, G. Gasaneo, and F. D. Colavecchia, Phys. Rev. A 89, 012713 (2014).

[33] R. K. Peterkop, Theory of Ionization of Atoms by Electron Impact (Colorado Associated University Press, Boulder, CO, 1977).

[34] A. S. Kadyrov, A. M. Mukhamedzhanov, A. T. Stelbovics, I. Bray, and F. Pirlepesov, Phys. Rev. A 68, 022703 (2003).

[35] A. S. Kadyrov, A. M. Mukhamedzhanov, A. T. Stelbovics, and I. Bray, Phys. Rev. A 70, 062703 (2004).

[36] G. Gasaneo, D. M. Mitnik, A. L. Frapiccini, F. D. Colavecchia, and J. M. Randazzo, J. Phys. Chem. A 113, 14573 (2009).

[37] D. M. Mitnik, G. Gasaneo, L. U. Ancarani, and M. J. Ambrosio, J. Phys.: Conf. Ser. 488, 012049 (2014).

[38] LAPACK-linear algebra package, http://www.netlib.org/lapack/ 